\title{
10. educational MULTIMEdia IN A NETWORK CENTRIC SOCIETY: REQUIREMENTS, ENABLERS AND NEGATIVE ASPECTS.
}

\author{
K.D. Levin \\ Tel Aviv College of Management \\ 7 Rabin Blvd, Rishon Lezion, Israel, \\ Phone: 972-3-9634267, Fax: 972-3-9634210 \\ email:dlevin@colman.ac.il
}

\begin{abstract}
The purpose of the paper is to identify the basic requirements for a network centric education and learning and to discuss the technological developments that enable the applications of educational multimedia over the network. Some of these applications will be reviewed as well as the problems ("balkanization", inequality and economical cost) associated with the positive and the negative impact of the network centric society.
\end{abstract}

\section{Keywords}

Distance Learning, Home Learning, Multimedia, Videoconferencing 


\section{Introduction}

As education and learning moves beyond the recall of facts and into the area of creativity, problem solving, analysis and evaluation, learners require inter personal communication in order to question, challenge and discuss (Bates 1994). With the explosive growth in Internet connections worldwide, networked communication has the potential to shrink geographic distances and facilitate information exchange among people of various backgrounds.

There are positive and negative outcomes to these developments. Information and communication technology can link geographically separated people and help them locate interesting or compatible resources. Although these attributes have the potential to bridge gaps and unite communities, they also have the potential to fragment interaction and divide groups by screening out less preferred contact.

In section 2 we will identify the basic requirements for a network centric education and learning. In section 3 we will discuss the technological developments that enable the applications of educational multimedia over the network. In section 4 we will review some of these applications. The negative impacts of "balkanization" (Alstyne and Brynjolfsson 1997), inequality and economical extranalities will be discussed in section 5. Section 6 concludes the paper by identifying problems for further research.

\section{Basic Requirements}

Regardless of the model of pedagogy that one works with, interaction between instructor and student and among students is central to the process. Traditional distance learning programs have most often replicated the teacher lecture model. These environments primarily utilize one-way transmission of video signals, with limited student feedback (via telephone calls). Interaction between the teacher and students is limited and interaction between students at different sites is non existent.

The network centric educational context will need to encompass the following:

- Support for individual work, interacting with learning material that may be available locally or remotely.

- Support for collaborative work both locally and at different remote sites, either asynchronously or synchronously, and most likely in a multimedia form.

- Support for a student role guided by an instructor.

- Support for an instructor role supervising and guiding the students.

The Interactive Multimedia Distance Learning (Wilson and Mosher 1994) is a good model for the "virtual classroom" (prototype by Rensselar and AT\&T) that combines rich communications' capabilities of 2 way, real time, video and data sharing among students and teacher. 
In traditional classrooms, instructors use text, graphs, animation, videos, sounds or live demonstrations to present curriculum content to students. They create exercises for students to work in a shared space (the blackboard), and they can write comments on student's work. Students are taking notes and can work on exercises locally on their desktop as well as on the shared blackboard. They can collaborate with other students and may alert the teacher that they would like to ask a question. The instructor is the leader of the class and may pass control to students in order to present their work or to ask questions. However, only the instructor may pass control and can take it away from the students. The basic features of the traditional classroom should be supported in a network environment in order to maintain a class structure. These features require:

- One workstation configured to be the leader. The leader can pass the focus to a participating workstation.

- Interconnected workstations configured to follow the actions of the leader.

- Events sharing mode were text, graphics, video or audio is presented to the workstations. When the focus is passed from the leader to the student's workstation, the students control the flow of shared events and everyone may see or hear the student's presentation.

- Each workstation can work independently.

- Hands raising were the students send a message to the instructor's workstation, letting the instructor know that they have a question. The instructor may choose to acknowledge the question or to respond to it at a more convenient time. When the instructor answers the student the focus may be passed to the student's workstation so others can view the discussion in an event sharing mode.

- Instructor pointer were the instructor can move a pointer around the students screen to emphasize important points in the information display.

In this environment, the instructor may use the lecture mode when appropriate, or to interact with the students performing work on their workstations. Students may share their work with other students and can engage in multiple discussions were control of the session is passed back and forth from instructor to student.

\section{Technological Enablers.}

Multimedia in education is developing fast due to the convergence of different media (text, data, graphics, video, audio) into a common digital platform. However, while stand-alone applications of multimedia will continue to be important in education, a much more significant development will be the application of high speed multimedia networks for educational purposes.

Software, hardware and content providers are struggling to provide standardized multimedia solutions, but the co-existence of numerous media standards and the rapid proliferation of these technologies presents problems. In an effort to resolve 
these conflicts, Netscape Communications', Progressive Networks and 40 other companies agreed on a proposed standard communications protocol for the realtime delivery of multimedia information over the Internet. The Real Time Streaming Protocol (RTSP) addresses issues such as fidelity, packet loss, latency and reliability. PC technology is beginning to impact the entertainment, education and communications' industries as companies begin to leverage the performance and features.

The convergence of multimedia capable computers, user friendly environments and relatively fast transmission technologies have led to an acceptable on-line experience for the average consumer. The Internet experience today has been largely constrained by limited bandwidth. There are times when getting information over the web justifies the humorous reference to the WWW as the "World Wide Wait".

An indication of this converging of computers, cmunications, content, and consumer electronics is a new standard being considered by the World Wide Web consortium called "Synchronized Multimedia Integration Language," that offers to enhance both the old (television) and the new (Web). SMIL enables authors to bring television-like content to the Web, avoiding the limitations of traditional television and significantly lowering the bandwidth requirements for transmitting this type of content over the Internet (details at http://www.w3.org/Press/SMIL).

As the Internet access marketplace continues to mature, telephone carriers, cable companies and others are racing to evaluate and deploy high bandwidth technologies in homes and in businesses.

Even with a vast increase in the bandwidth of the Internet, optical media will be able to deliver a significantly better multimedia experience for the future. Educational multimedia applications should integrate both technologies. They should be delivered on the Internet while high quality multimedia experience will be delivered on optical media, augmenting and enhancing the on line (Internet) version of the product.

What is emerging is a highly connected network providing access to sources, tools and information across disciplinary, institutional, and national and international boundaries. It will soon be difficult to imagine a course not on the Web. However, the challenge is to deal with the technologies at hand, which are quickly outdated, while not being overwhelmed by the new and exciting opportunities available and promised.

\section{Network Centric Educational Applications}

The Web-based education market is showing continuous growth in terms of the number of programs, the number of venues and the number of certified people. Lotus and Microsoft are playing a leading role in using the Web as a medium for the delivery of training material. Web-based training makes bad instruction impossible because such factors as poor instructors or class room logistics cannot affect the training. However, inspiring lecturers are absent in Web training, even though students can ask questions and receive answers over the Web. 
The Interactive Distance Education Alliance (IDEA) proposal is a distance learning solution that was presented to several large companies, including Intel and TRW. The study determined that there was a high demand for distance programs in business and engineering, and focused on interactive teleconferencing and Webbased delivery. The study showed that faculty could adapt to the electronic classroom model quickly, given appropriate technical support. The IDEA proposal was presented to several companies, with two firms, Intel and TRW, agreeing to fund initial study projects beginning in January 1996.

The Global Information Network in Education (GINIE), became operational in early 1996. GINIE, funded by UNESCO and the US Agency for International Development, serves as a multi-channel information network to collect, analyze and disseminate information of use to policy-makers, educators, government and agency officials and local communities. The network also provides education information to nations in crisis, such as Bosnia-Herzegovina, the People's Republic of China, and others.

Pennsylvania's Education Network (PEN) is not just for Pennsylvanians any more. The network's Digital Object Repository (DOR) will store curriculum frameworks and components in a variety of multimedia data types, including video, audio and images. PEN-DOR is a Technology Testbed Project funded in part by Pennsylvania's Link-to-Learn program. Its purpose is to provide Pennsylvania schools, libraries, and communities with virtually unlimited access to the information available on global networks. Its purpose is to provide multimedia education materials to the Keystone State's teachers and students over the World Wide Web. The system, which can expand to meet growing needs, will be integrated into the Global Information Network in Education (GINIE) and eventually will be accessible to students and educators worldwide via the Web.

Colleges and universities throughout the US are increasing their on-line-based course offerings, allowing working students to receive the benefits of a secondary education from the convenience of their home. Many students physically unable to attend colleges because of work-duty or geographic locations are thus able to earn business degrees they ordinarily would not receive. Duke University, UCLA and UC Berkeley each offers diverse business curricula through their on-line services, and students can easily form study groups with fellow classmates through on-line chat rooms and contact instructors via E-mail.

Thanks to the Internet, credentials from UCLA are just a mouse click away from evening classes at your local community college. You can take courses at your own convenience and have the worldwide library of the Web at your fingertips for instant research. The cost for taking on-line courses is comparable with physically attending class, but without transportation, accommodations, and other sundry costs. More important, in response to the mushrooming number of small businesses, universities today are offering a variety of entrepreneurship cybercourses. Here's a sample of on-line business courses offered by major universities.

- Duke University's Fuqua School of Business's Global Executive MBA offers access to Fuqua's MBA program via the Internet (www.fuqua. duke.edu).

- The University of California Los Angeles recently opened up its extension classes to the Internet through a partnership with The Home Education Network (www.then.com). 
- Florida-based Open University offers a truly virtual campus with a series of business classes available exclusively on the Internet (www.openu.com).

- New York City's New School for Social Research provides on-line education through its Distance Instruction for Adult Learning program (www.dialnsa.edu).

The U.S. Government, with assistance of public and private organizations, steadily implements policy in cooperation with the former USSR, in the areas of education, business, economics and culture. California State University, Los Angeles is reaching out to build more academic bridges in the world. Their Center for Newly Independent States Studies (CNISS) contributes to the strategic goals of building and securing educational, business and cultural links between the U.S. and NIS countries.

Other initiatives at the international arena include the followings: Australia Technical and Further Education institutes will provide 30 percent of their education programs over the Internet. Japan - Nippon Telegraph and Telephone Corp. New videoconferencing system "Konet Plan," is intended to bring Japanese school teachers and students together using both videoconferencing and the Internet. Canada - Stentor, an alliance of Canadian telephone companies, is financing a 10 year initiative, called Beacon, that will bring broadband, multimedia services to $80 \%$ of all home and businesses in Canada by the year 2004 .

\section{The Negative Aspects}

Over the last several decades, there has been a decline in both civic engagements and in the norms, trust and features of social life needed to achieve social objectives. Since 1965, the amount of time Americans spend in local clubs is down by one half. Attendance at town meetings is down $39 \%$. The number of people working for political parties dropped $56 \%$..

The general argument is fairly simple Internet users can seek out interactions with like-minded individuals who have similar values, and thus become less likely to interact with people whose values differ from their own. If IT provides a lubricant that allows for contact that is more focused than contacts available locally, then, local heterogeneity can give way to virtual homogeneity as communities coalesce across geographic boundaries.

With increased connectivity and bandwidth an emerging global village represents one possible outcome from a range of possibilities. It is also possible that improving communication's access through emerging technology will fragment society and introduce balkanization of preferences (Alstyne and Brynjolfsson 1997), social, intellectual and economic affiliations, analogous to geographic regions. Just as separation in physical space can divide geographic groups, separation in virtual space, or "cyberbalkanization" can divide special interest groups. Several virtual communities based on identity derived from racial origin [NetNoir$\mathrm{http}: / / \mathrm{www} . n e t n o i r . c o m / i n d e x . h t m l]$, geographical location [Blacks-burg Electronic Village-http://www.bev.net] and social issues [Public Electronic Network- 
http://pen.ci.santa-monica.ca.us], have appeared in recent years. These virtual communities, serve to satisfy the needs of the users based on commonalties. They can be broadly categorized as communities of relationship, communities of interest, communities of transaction, and communities of fantasy (Armstrong and Hagel 1996).

At some point, almost everything needed for teaching and learning will be provided on the Internet: textbooks, encyclopedias, magazines, newspapers, library books, multimedia software, research databases, photos, videos, and more. Most of it is not free. Valuable and substantive educational resources cannot be researched, developed, tested, and disseminated without cost. It takes a great deal of effort to develop valuable material. That effort must be compensated in order to continue. It can cost as much as $\$ 1$ million to produce an educational CD-ROM, and even more to publish a textbook. The sooner we all recognize the fallacy of free education on the Internet, the sooner publishers and developers will be willing to take the risk of embracing the Internet for delivering quality instruction.

The result of a recently completed RAND study on the distribution of information and communication capabilities across the US populace indicates that since 1989 (and even before), gaps in access to computers and networks have increased significantly over time as a function of income. The gap between the information rich and the information poor can widen with virtual communities (Alstyne and Brynjolfsson 1995) and there should be no illusions that a greater sense of community will inexorably result. The small computer finally gained classroom acceptance, though, largely due to parent lobbying and aggressive advertising by manufacturers. While many believe today that computers, unlike radio and TV, may qualitatively change education, there is a growing apprehension that classroom IT has widened the gap between the have and have-not.

Operation Social Development (OSD) was established by a coalition of Israeli high technology industry leaders in cooperation with Israeli government. The ground breaking project, A Computer for Every Child, supplies disadvantages children from all backgrounds with state of the art home computers, top quality educational software, access to the Internet and comprehensive user instruction. Even more powerful than placing computers in schools - where too little time and too few facilities limit usage - A Computer for Every Child gives disadvantaged youth direct, unlimited access to their own PC.

Critical to the success of the project is the 60 hours of required instruction in computer hardware and software. Topics include use of the Internet, electronic mail, access to databases and programs which aid in the research and presentation of school papers and projects. After successfully completing the training course, the children receive the full package of educational programs on CD-ROM's. All programs are approved by the Israeli Ministry of Education and are used by most Israeli schools.

\section{Conclusions}

Electronic communications have become increasingly important to the conduct of higher education. The new digital technologies are changing the way instruction is 
delivered. In this paper we identified the basic requirements for a network centric education and learning and discussed the technological developments that enable the applications of educational multimedia over the network. There are positive and negative outcomes to these developments. Information and communication technology can link geographically separated people and help them locate interesting or compatible resources. Although these attributes have the potential to bridge gaps and unite communities, they also have the potential to fragment interaction and divide groups by screening out less preferred contact.

The following summarizes some of the issues discussed in this paper:

- We are breaking with the "credit for contact" model and considering alternatives to lecture as a delivery mode. Students are not place-bound or timeconstrained.

- Students are developing new skills to access, organize and synthesize information received via the Internet.

- Teachers need incentives to incorporate technology into their educational programs. They must see benefits to their own teaching and to their students' learning.

- A new role for the teacher has been identified, one of facilitator and coach, thus establishing a new relationship between teacher and learner.

What is emerging is a highly connected network providing access to resources, tools and information across disciplinary, institutional, and national and international boundaries. It will soon be difficult to imagine a course not on the Web. Yet in spite of those major advances in networking technology, there are important applications that faculty and students can not pursue. Learning networks should promote acquisition and knowledge building to enrich locally available resources. However, the challenge is to deal with the technologies at hand, which are quickly outdated, while not being overwhelmed by the new and exciting opportunities available and promised.

Further work needs to be done to study the pedagogical, economical and sociological implications of network centric education. Some of these issues are:

- What kinds of class activities are best performed locally on individual work station and what activities are best performed through interactions over the network.

- How to assure the privacy of the student and the confidentiality of data?

- How to provide sufficient compensation to providers of classroom activities? How to finance this compensation?

- How can we guarantee that humans, not computers, are still identified as the instructor? 


\section{References}

Alstyne, M. and Brynjolfsson, E. (1995) "Communication Networks and the Rise of an Information Elite - Do Computers Help the Rich get Richer?" Proceedings of the Sixteenth International Conference on Information Systems, 1995, pp. 93-96.

Alstyne, M. and Brynjolfsson, E. (1997) "Electronic Communities: Global Villages or Cyber Balkanization?", Proceedings of the Seventeenth International Conference on Information Systems, 1997, pp. 373-379.

Armstrong, A., and Hagel III, J. (1996) "The Real Value of On-line Communities," Harvard Business Review (74:3), May-June 1996, pp. 134-141.

Bates. A.W. (1994) "Educational Multi-Media in a Networked Society", Proceedings of EDMEDIA 94, pp. 3-8.

Jordan, E. and Hewett, W.G., (1997) "Discussion Group: Teaching and learning using the WWW", The Place of Information Technology in Management and Business Education, pp. 219-230, Chapman and Hall, 1997.

Wilson,J.M. and Mosher,D.N., (1994) "Interactive multimedia Distance Learning (IMDL) The Prototype of the Virtual Classroom", Proceedings of ED-MEDIA 94, pp.563-570.

\section{Biography}

K. Dan Levin is currently a senior lecturer at the College of Management. He was a senior Lecturer and the head of the information system program at the faculty of Industrial Engineering \& Management, Technion, the Israeli Institute of Technology and an associate professor of decision sciences at the Wharton School, University of Pennsylvania. He has over 30 years of experience in the high technologies sector. Specialization in the political, technological and market forces operating at the intersection of computers, communications and image processing. 